\title{
New Sustainable Systems Engineering Program PROPOSAL - MiNDSET AND DEVELOPMENT
}

\author{
Robyn Paul, Gillian Ayers, Joule Bergerson, Kerry Black, Tanya Brucker, Caroline Dawoud, Marjan \\ Eggermont, Andy Knight, Sean McCoy, Elena Rangelova, David Wood \\ University of Calgary \\ rmpaul@ucalgary.ca
}

\begin{abstract}
With the continued climate crisis, there is increasing recognition for the important of sustainability education in engineering. At the University of Calgary, we are developing a program in Sustainable Systems Engineering to address this need. Systems thinking and sustainability are intrinsically linked, as in order to comprehend the wicked challenges of sustainability today, we must take a holistic, interconnected, systems approach. This paper outlines sustainability education literature, and our approach to program development. Overall, we hope to foster mindsets and develop engineering students who are able to fundamentally shift the discourse on sustainability engineering within industry, and critically reflect on the role of engineering itself.
\end{abstract}

Keywords: Sustainability : Sustainable Engineering : Systems Thinking : Regenerative Design : Transdisciplinary Engineering : Program Development

\section{INTRODUCTION}

Across engineering education in Canada and the world, there is an increased recognition of the importance of sustainability mindsets, particularly since the adoption of the Sustainable Development Goals in 2015 [20]. Traditionally, sustainability within engineering has focused on the technical issues and taken a very end-of-theline approach, for example looking at how to technically manage and reduce pollution [19]. There is a growing recognition of the need to transition to a more holistic systems approach to more effectively address sustainability by considering the "complex, systemic interconnections and cause and effect relationships" [16]. At the University of Calgary, we have a unique opportunity to develop a program in sustainable systems engineering from the ground up, where we intentionally imbed a systems approach and a regenerative design mindset from the onset.

The Sustainable Development Goals [20] have been helpful as a starting point, and we have taken these a step further by using additional progressive and pioneering theories and frameworks to guide our development which are embedded with mindsets in systems thinking and regenerative design. For example, the engineering for one planet framework [7] aims to "minimize negative impacts, strive to achieve at least net neutral outcomes, and, ideally, [ones that are] are restorative" (p.9). The development of the sustainable systems engineering program also integrates social justice (ex. Common good matrix [5]) and economics (ex. Doughnut economics [3]). These sociocultural and economic elements of sustainability are two essential pillars of sustainability that are often still overshadowed by the techno-environmental perspectives [19], which leads to limited depth of comprehension of the ethical issues that are inherent to engineering and technical progress.

This paper provides an overview of the mindset behind the development of the sustainable systems engineering program at the University of Calgary. Specifically, we discuss the systems thinking mindset and how this is integrated across our pedagogical approach in terms of how students learn and develop when thinking about systems. We provide a brief overview of the sustainable design spine that spans across all years and aims to connect the content taught across all courses from a systems perspective. Additionally, we will provide background on the motivation for the program, including different perspectives on sustainability and stakeholder engagement.

Overall, we hope to foster mindsets in order to develop engineering students who are able to fundamentally shift the discourse on sustainability engineering within industry, and critically reflect on the role of engineering itself. As engineers our responsibility is not only to society and culture, but also to nature and the planet. We must take accountability for the significant damage the engineering profession has contributed to the climate crisis and consider what role engineering plays in the transition away from fossil fuels. This program aims to provide students with the necessary skillsets and mindsets to foster real change across engineering industries to better support the interrelated elements of our society and planet.

\section{BACKGROUND}

\subsection{Sustainability in Engineering Education}

In this section we provide a summary of scholarly literature on sustainability in engineering education. Since 
2010 and particularly since 2015 with the adoption of the SDGs across many countries, discussions around sustainability in engineering have continued to increase. A 2019 literature review looked at patterns and trends in engineering education in sustainability [19]. Through analyzing papers from 2001 to 2017 , the authors found that keywords on transdisciplinary and collaborative networking have seen a particular increased, almost tripling in frequency in the last five years.

In their analysis they also looked at the three pillars of sustainability: techno-environmental, techno-economic, and socio-cultural. As sustainability literature was increasing between 2008-2012, the highest increase was seen in the techno-environmental pillar, whereas between 2013-2017 a larger increase was seen in the other two pillars (economic and social) [19]. These results show how over time we've realized in order to achieve the desired environmental outcomes, we need to address the economic and social issues of sustainability.

Education for sustainability can be defined as an approach to education that "encourages individuals to develop skills to critically enquire and systematically think about problems in ways that allow them to explore the associated complexity, and the implications for a more sustainable way of being" [9, p.133]. Sustainability education must not only be a separate, silo-ed course (or perhaps even program), but rather needs to be integrated across all areas of the curriculum in order to foster an interdisciplinary approach.

There are a wide variety of competencies required for sustainability education, as summarized by [16] these include: interdisciplinary skills, critical thinking, working with multiple stakeholders, foresighted thinking, dealing with complexity and uncertainty, social justice and equity, care for the environment and physical world, and a big picture perspective. Specifically, they argue that "systems thinking can be viewed as the keystone that underpins those [competencies] identified in the literature" [16].

\subsection{Sustainability is Intrinsically Systems- Based}

How to better educate engineers for sustainability has been increasingly studied over the past couple decades. Due to the complex nature of sustainability problems, often called 'wicked' problems, there is an essential need to understand the bigger picture, the "complex, systemic interconnections and cause and effect relationships" of issues like climate change [16].

Many scholars support this perspective, arguing that sustainability issues are "intrinsically systems problems" [13] and students must learn to "think and act systematically" in order to address the complex, wicked problems of sustainability [12]. Others have also discussed how systems theory is essential to sustainability learning and that only through considering systems can we effectively make change for sustainability [14].
Systems thinking at its core is about considering complexities of the world based on the big picture and relationships, rather than breaking it down into isolated parts [15]. Sandri has argued that systems thinking is a threshold concept for sustainability, where students' ability to engage with and understand systems thinking is directly related to their ability to comprehend sustainability [16].

It is therefore important when thinking about teaching sustainability to consider how to most effectively teach for systems thinking. The epistemological approach for teaching systems thinking must go beyond simply presenting content and providing opportunities for problem solving [16]. Rather, it is important for teachers to consider epistemic development and metacognition as a key foundation required for students' ability to learn and adopt a systems thinking approach [16].

\subsection{Deconstructing Hierarchical Thinking for Sustainability Learning}

For students to grasp the concept of sustainability, it is important that we deconstruct the hierarchies that exists within engineering education. For example, the emotionalrational dualism assumes an engineer should be a "rational problem solver who wants to do the best for society from a utilitarian perspective" [11]. Often in engineering classrooms professors tend to emphasize and value higher the importance of rational decision making above supporting students who are able to use their emotions and feelings to critically make decisions and solve problems [10]. This perspective assumes that engineering exists within a meritocracy and that our engineering education is value-neutral [2].

In reality, particularly in sustainability engineering education, in order to solve complex problems such as climate change, it is essential that we deconstruct this dualism and integrate the emotional side of learning and acknowledge multiple ways of knowing. This dualistic thinking engrained within engineering culture also maintains a hierarchical relationship where we see humans as rational and superior to those who are not human [1]. Humans are seen as superior entities and non-human are seen as lower-value entities which are not rational and therefore silent and unintelligent. To engage in transformative sustainability education, we therefore must breakdown this mindset and acknowledge "the more-thanhuman as agential and communicative beings" [1].

Rather than valuing emotions as separate knowing, we must foster a sustainability education with connected knowing where our emotions are integrated in our critical thinking and problem solving [11]. We must support our students in surpassing the necessary threshold concepts (troublesome, transformative, and irreversible knowledge gains) such as understanding that knowing is relational, where our knowledge is co-constructed through our relationships and interactions with humans, ideas, cultures, and other-than-human beings [1]. 
Overall, before students can deeply engage with transformative sustainability education, we must foster an understanding of the power of dominant beliefs. These belief systems, ideologies, and hidden curriculum undermine a students' ability to see other ways of knowing as valid, and thus we must address this to support students in becoming more open to concepts within sustainability engineering education [1].

\subsection{Existing Programs in Canada}

On the list of accredited engineering programs in Canada, there are three accredited programs which include the word "sustainability" (one at UPEI and two at Carleton) [6], and one program recently having graduated their first cohort of students (Simon Fraser). There are also many additional programs which include names such as "environmental" and "green". As part of our development process, we have met with multiple individuals from programs across Canada in order to better understand their mindset and approach to sustainability education.

\section{APPROACHES TO SUSTAINABILITY IN ENGINEERING}

The authorship on this paper shows the diversity of perspectives which we are bringing to the development of this Sustainable Systems Engineering program. It was essential when we started this process, we were bringing an interdisciplinary perspective from a wide range of engineering disciplines. The program development committee is made up of seven faculty members from across five engineering faculties (including both teachingstream and research-stream faculty), two staff members, and two students (one undergraduate and one graduate student). For that reason, we wanted to highlight some of our own perspectives and approaches to sustainability. Each of these perspectives are brought to the discussions to provide a holistic and diverse view on sustainability education. The following shows three examples from faculty members on the program development committee.

\subsection{Renewable Energy Technology}

From my perspective, the value of the initiative to develop the sustainable engineering program is the context it provides for my research and teaching area of renewable energy technology. Currently, I teach hydro, wind, and solar technologies followed by battery storage and system integration. The emphasis is on the technology and the context is given primarily in economic terms - the fact that wind and solar are now the cheapest forms of electricity generation in resource-rich Alberta. In other words, the sustainability framework is largely implied and the concentration on electricity production leaves out many energy sectors. Society is moving to the integration of these sectors and engineering education must as well. This is crucially important for decarbonization and its possible pathways through the hydrogen economy, but difficult to achieve in a conventional engineering program and other types of generators - geothermal, marine etc - are left out and the crucial aspect of energy systems modeling is not addressed at all.

\subsection{Geomatics Technologies}

Many sustainable systems rely on geomatics technologies to collect and process geospatial data in support of sustainable development and design. These technologies encompass geospatial information systems, remote sensing from satellites, unmanned aerial systems and terrestrial sensors, global space-borne Earth observations systems, location services, web maps and mobile mapping, among others. All these technologies provide accurate, precise, timely, seamless and critical information on local, regional and global scales of the natural and built environment. The spectrum of sustainable development applications using geomatics data is broad: (1) environmental impact assessment (renewable energy sources, soil and water consumption, soil erosion, and water pollution); (2) disaster monitoring, preparedness and mitigation; (3) risk management and spatial decisionmaking systems; (4) urban development processes (spatial and temporal changes in land use and connection to climate changes, changes in the natural, built, and social environments); (5) sustainable land management (integration of land, water resources, biodiversity and environmental management); (6) resource development and environmental protection; (7) rural-urban land integration and sustainable communities; and (8) smart cities. A few of the principles and goals of sustainable development are explored in the geomatics engineering program, mainly in geospatial information system projects. In the third year, the main focus is on remote sensing and global Earth observation systems with themes such as groundwater depletion and monitoring of water mass balance, flood and drought monitoring and preparedness, air pollution monitoring from space, global and local sea level rise and disaster preparedness, web maps. A sustainable community development project is completed by part of the fourth-year geomatics students.

\subsection{Circular and Regenerative Systems}

Our current success is largely on the back of ecosystem services and natural capital being transformed into other forms of capital ever since the early days of the Industrial Revolution.

Today our model of economic growth continues to be largely based on this unsustainable linear approach. If we don't appreciate the crucial role that natural capital plays for our economic success (a recent number valued it at $\$ 125$ trillion per year), we will under-manage natural capital and our planet's ability to regenerate itself and to provide that natural capital, and we will deny future 
generations of future economic success and a healthy planet for all life.

Our economy has grown exponentially, adding billions of people and quickly depleting the very same natural capital on which it thrives--from the shortage of fresh water, from the loss of phosphorus, to the pollution of oceans, soil degradation, and biodiversity loss to name just a few [18].

Canada's overshoot day is March 14 this year - the date on which Earth Overshoot Day (currently on August 22) would fall if all of humanity consumed like we did [4].

We can move to a society with abundant clean energy, and a cradle-to-cradle material bank.

We can build a circular material system where materials are either used over, and over, or are safely returned to the biosphere: a circular system of biological and technical nutrients. Most materials can be used repeatedly, as long as they are kept pure, as long as they are designed for disassembly, repair, and reuse, applying better design principles. France, in January of this year, began requiring makers of certain electronic devices, including smartphones and laptops, to tell consumers how repairable their products are. Manufacturers selling these devices in France must give their products a score, or "repairability index," based on a range of criteria including how easy it is to take the product apart and the availability of spare parts and technical documents [17]. The UK is introducing a 'right to repair' law this summer [8]. We can teach our students in addition about environmentally friendly materials, about ethical supply chains and design for dignified employment, ideas for maintenance, modularity, mono-material, and zero-waste.

Can we also, as Martin Stuchtey calls it, move to a highproductivity regenerative system [18]? This would require high-performing systems, which the new program aims to prepare students for. Some examples are systems of higher utilization of resources through sharing, virtualization of activities, so that we're avoiding products in the first place, better integration of products into systems-- mobility systems, food systems, built-environment systems-- and less wastefulness overall. This can result in economic opportunity and exciting challenges for our future engineering students.

\section{Program DeVelopment}

\subsection{Main Program Goals}

Driving the program goals comes from the importance of systems within sustainability engineering. Figure 1 shows our underlying mindset of aiming to teach sustainability from a life-centered design perspective. Our current working definition of sustainability systems engineering:

Sustainable Systems Engineering is a technical, life-centered, transdisciplinary program focusing on

CEEA-ACEG21; Paper 140

University of Prince Edward Island; June 21 - 23, 2021- 4 of 7 - how to design, integrate, and manage complex systems over their life cycles with the goal of environmental, economic, and social sustainability.

We continuously iterate on this definition as we progress through the program development process and engage in discussions with stakeholders.

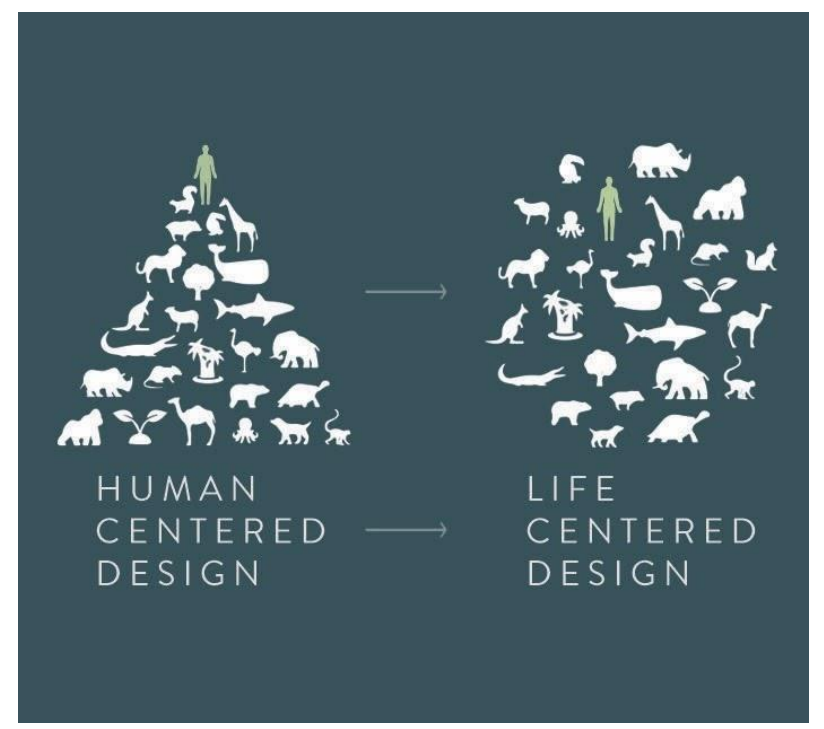

Fig. 1. Human-centered design (hierarchical) vs. lifecentered design.

Our learning outcomes for the program are iterating from a foundation which is based off The Engineering for One Planet Framework [7]. Systems thinking sits at the middle of our learning outcomes, as it is central, and the most fundamental learning outcome required for all sustainable engineering practices. The One planet Framework specifically defines systems thinking as:

Systems thinking is a critical approach for engineers to understand that designs rely upon and exist within systems, to identify the impacts and influences of the different and interconnected environmental, economic and social factors of the design system and to recognize that their designs themselves are systems. [7, p.10]

Surrounding this central learning outcome of systems thinking are additional outcomes including critical thinking, social responsibility, community \& teamwork, along with others. See figure 2 below for more details. From this base framework, we have continued to brainstorm and develop our program learning outcomes. 


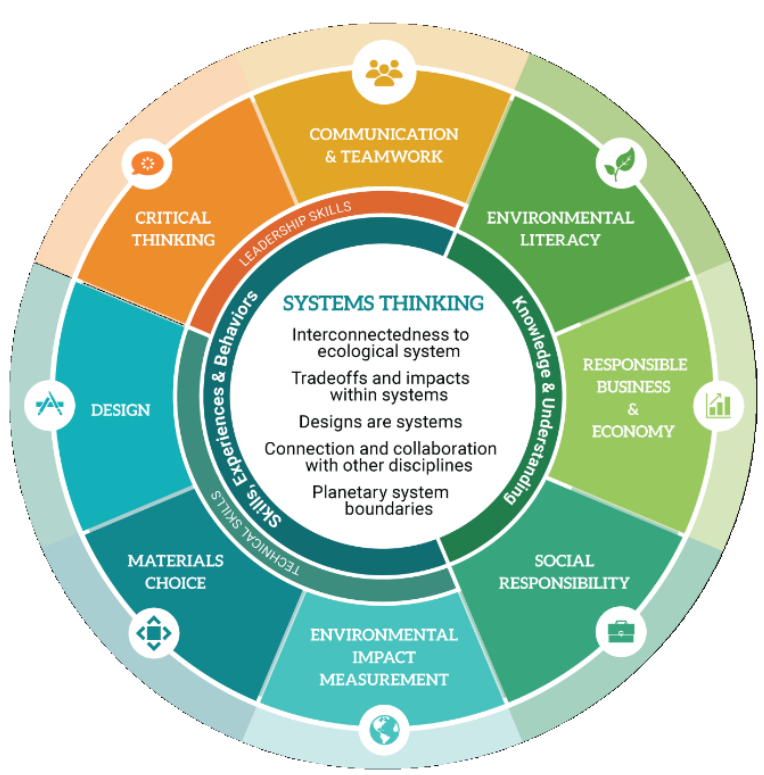

Fig. 2. The Engineering for One Planet Framework [7]

\subsection{Current Program Design}

Although the curriculum is still under continued iteration and development, there are a few key features within the curriculum to highlight.

First-year will follow the common first-year format that exists at the University of Calgary. This common first-year provides students with an introduction to all of our programs, where they make their program choice about two thirds of the way through their first-year of engineering. Recently, changes have also been made to the first-year design course where there is an added emphasis on exploring sustainability within their first-year design project.

The program curriculum is built on an interdisciplinary framework where courses from programs across the school of engineering support the introduction and development of foundation engineering knowledge. This includes integrating a wide variety of courses from civil, energy and environment, mechanical, geomatics and electrical engineering. For example, see in Fig. 3 a draft of the thirdyear curriculum which includes courses from ENCI (civil), ENME (mechanical), ENEL (electrical), ENGO (geomatics), and ENEE (energy and environment).

Across all years there will be a design spine, where students learn fundamentals of systems design for sustainability, including integration of economics, design for learning from natural systems, systems and society in sustainability design, and indigenous peoples within sustainable engineering development. Much of these sustainability design spine courses are being developed in alignment with Engineers Canada guidelines on sustainable development and environment stewardship for professional engineers [21]. Examples of new courses are System of Systems Modelling and Analysis, Sustainable Entrepreneurship and Social Innovation, Bio-inspired Design, and Principles of Sustainability and Indigenous Knowledge Systems.

Between second- and third-year, students will engage in a 2-week intensive field course, Northern Sustainable Systems combined with Sustainability Research Methods, with options for location including the Kluane Lake Research Station (located $220 \mathrm{~km}$ northwest of Whitehorse, Yukon) and W. A. Ranches at The University of Calgary (Rocky View County, Alberta).

In their final year, students will be able to mix and match courses from different engineering programs and technical electives within the following themes:

- Sustainable systems for Environment

- Sustainable systems for Communities and Cities

- Sustainable systems for Energy and Resources

- Sustainable systems for Food, Agriculture, and Biomass

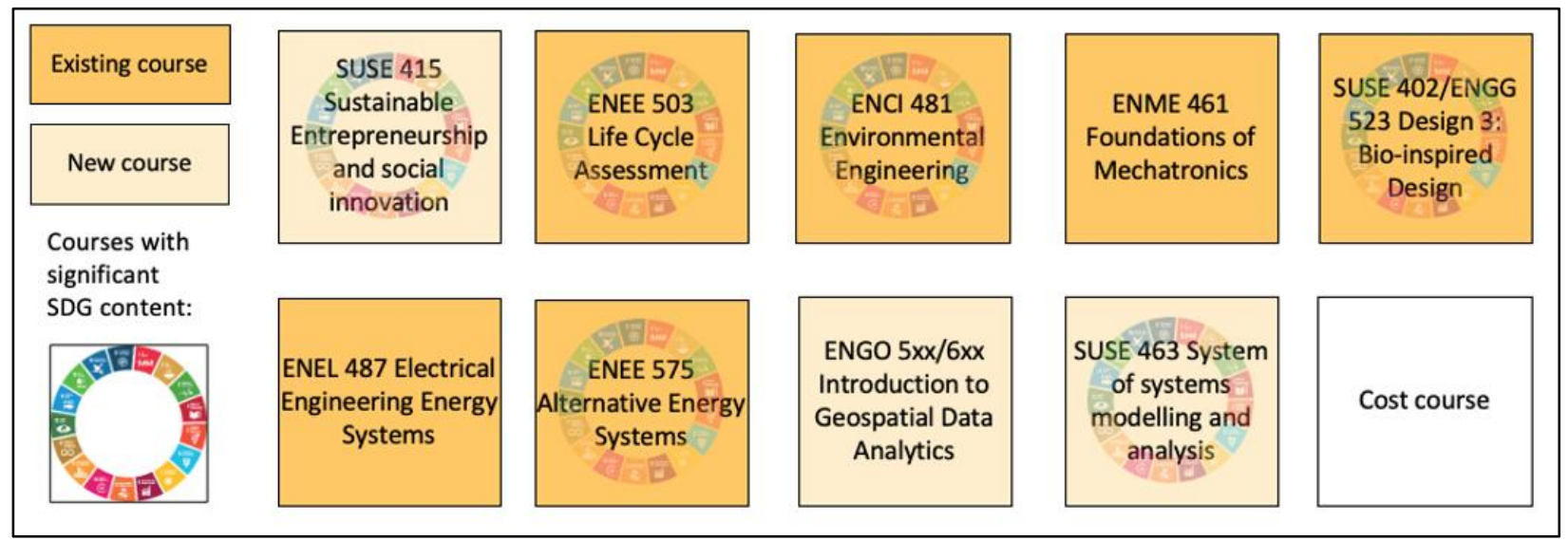

Fig. 3. Draft third-year curriculum for Sustainable Systems Engineering, which shows the interdisciplinary nature of the program, integrating existing courses from across multiple engineering disciplines. 
Within each of the themes, there will also be new courses developed on topics such as energy systems modelling, distributed energy sources/remote systems, industrial ecology, and other special topics in sustainable systems.

Overall, the curriculum is in continued flux and development. We hope through sharing our approach and our current curriculum considerations that we are able to engage in discussions with the Canadian engineering education community to further improve sustainability education across this country.

\subsection{Program Self-Assessment}

In addition to $\mathrm{CEAB}$ continual improvement measures, we will be using a program sustainability assessment tool. This tool looks at the ability of a program to maintain programming and benefits over time. The tool looks at 8 categories that influence a programs sustainability over time. Examples of categories are: Environmental support, a category that assesses the internal and external climate of support for the program. Partnerships and organizational capacity, program evaluation and adaptation, and communication and strategic planning are additional components that will be monitored using this tool. Each category includes and additional 5 questions that affect program sustainability, for example "The program makes decisions about which components are ineffective and should not continue" and "The program has the capacity for quality program evaluation" [22].

\section{CONCLUSIONS}

To address the climate crisis of today, we must be training future engineers who are prepared to make the necessary changes. It is essential that we approach sustainability engineering education within a systems framework, where through our pedagogies and curriculum, we actively deconstruct the cultural barriers to sustainability in engineering. This paper provided a literature foundation for sustainable systems engineering and an overview of our current program development progress. We hope to engage in discussions and feedback on the program and collaborate with institutions from across Canada in order to best support our students in becoming engineers who are equipped with the skills to advocate for a better future world.

\section{References}

[1] M. J. Barrett, Matthew Harmin, Bryan Maracle, Molly Patterson, Christina Thomson, Michelle Flowers, and Kirk Bors. "Shifting relations with the more-than-human: Six threshold concepts for transformative sustainability learning." Environmental Education Research, vol. 23, no. 1, pp. 131-143, 2017.

[2] Erin A. Cech. "The (mis) framing of social justice: Why ideologies of depoliticization and meritocracy hinder engineers' ability to think about social injustices." In Engineering education for social justice, pp. 67-84. Springer, Dordrecht, 2013.

[3] Doughnut Economics Action Lab. "Doughnut Economics." Retrieved from: https://doughnuteconomics.org/aboutdoughnut-economics

[4] Earth Overshoot Day. "Country Overshoot Days." Retrieved from: https://www.overshootday.org/newsroom/ country-overshoot-days/

[5] Economy for the Common Good. "Common Good Matrix." Retrieved from: https://www.ecogood.org/applyecg/common-good-matrix/

[6] Engineers Canada. "Accredited Engineering Programs in Canada by Institution." Retrieved from: https://engineerscanada.ca/accreditation/accreditedprograms/institution

[7] EOP frameowork. "Engineering for One Planet." Retrieved from: https://engineeringforoneplanet.org/

[8] Roger Harrabin. "Right to repair' law to come in this summer." BBC News. March 10, 2021. Retrieved from: https://www.bbc.co.uk/news/amp/business-56340077

[9] Sarah Holdsworth, Carina Wyborn, Sarah Bekessy, and Ian Thomas. "Professional development for education for sustainability." International Journal of Sustainability in Higher Education, 2008.

[10] Nadia Kellam, Karen Gerow, Gregory Wilson, Joachim Walther, and Joshua Cruz. "Exploring emotional trajectories of engineering students: A narrative research approach." International Journal of Engineering Education, vol. 34, no. 6, pp. 1726-1740, 2018.

[11] Johanna Lönngren, Tom Adawi, and Maria Berge. “ 'I don't want to be influenced by emotions'-Engineering students' emotional positioning in discussions about wicked sustainability problems." In Proc. IEEE Frontiers in Education Conference (FIE), 2020.

[12] S. Martin, and D. Morris. "Complexity, Systems Thinking and Practice: skills and techniques for managing complex systems." The Handbook of Sustainability Literacy-Skills for a changing world, pp. 156-164, 2009.

[13] Donella H Meadows. "Thinking in systems: A primer." Chelsea Green Publishing, 2008.

[14] Jenneth Parker. "Competencies for interdisciplinarity in higher education." International Journal of Sustainability in Higher Education, vol. 11, no. 4, pp. 325-38, 2010.

[15] M. Ramage and K. Shipp. "Systems thinkers." London: The Open University \& Springer. 2009.

[16] Orana Jade Sandri. "Threshold concepts, systems and learning for sustainability." Environmental Education Research vol. 19, no. 6, pp. 810-822, 2013.

[17] Maddie Stone. "Why France's new 'repairability index' is a big deal." Grist. February 8, 2021. Retrieved from: https://grist.org/climate/why-frances-new-repairabilityindex-is-a-big-deal/ 
[18] Martin Stuchtey. "A Good Disruption.” Produced by the Ellen MacArthur Foundation for the Disruptive Innovation Festival. 2017. Retrieved from: https://www.youtube.com/ watch?v=uT66CRYkSM8

[19] Gemma Tejedor, Martí Rosas-Casals, and Jordi Segalas. "Patterns and trends in engineering education in sustainability." International Journal of Sustainability in Higher Education, 2019.

[20] UNDP. "Sustainable development goals." Retrieved from: https://www.undp.org/content/undp/en/home/sustainabledevelopment-goals.html
[21] Engineers Canada. "National guideline on sustainable development and environmental stewardship for professional engineers." Retrieved from: https://engineerscanada.ca/national-guideline-onsustainable-development-and-environmental-stewardshipfor-professional-engineers

[22] Centre for Public Health Systems Science. "Program Sustainability Assessment Tool v2." Retrieved from: https://sustaintool.org/wpcontent/uploads/2016/12/Sustainability-ToolV2_wscoring_12.11.13.pdf 